\title{
Evaluation of Methanolic and Ethanolic Extracts of Azadirachta Indica Seed Oil for Hypoglycaemic Properties and Effects on Some Biochemical Parameters in Diabetic Mice
}

\author{
Muhammad HL., ${ }^{1}$ Kabiru AY., ${ }^{2}$ Makun HA., ${ }^{1}$ Adefolalu FS., ${ }^{2}$ Fasiku OV., ${ }^{1}$ and \\ Abdullah $\mathrm{AS}^{3}$ \\ ${ }^{I}$ Toxicology Unit, Department of Biochemistry, Federal University of Technology, Minna \\ ${ }^{2}$ Trypanosomiasis and Malaria Research Unit, Department of Biochemistry, \\ Federal University of Technology, Minna \\ ${ }^{3}$ College of Health Sciences, Usmanu Danfodiyo University Teaching Hospital, Sokoto
}

\begin{abstract}
Azadirachta indica commonly called Indian Lilac or Margosa is a folkloric medicinal plant that has been exploited over time for the management of several diseases. The methanolic and ethanolic extracts of the seed oil were investigated for hypoglycaemic effect in diabetic mice. Diabetes was induced in mice with 300 $\mathrm{mg} / \mathrm{kg}$ bodyweight of alloxan monohydrate intraperitionealy and mice with blood glucose concentration > 200 $\mathrm{mg} / \mathrm{dl}$ were considered diabetic. Diabetic mice in two groups were orally administered $200 \mathrm{mg} / \mathrm{kg}$ bodyweight of ethanol and methanolic extracts respectively for seven days. The blood glucose decreased in descending order during the course of treatment in a time - dependent manner. Serum activities of alanine aminotranseferase $(20-100 \mathrm{u} / \mathrm{L})$, aspartate aminotransferase $(18-61 \mathrm{u} / \mathrm{L})$, and alkaline phosphatase $(42-140 \mathrm{u} / \mathrm{l})$ increased significantly $(p>0.05)$ compared to values for the control. The Serum levels of total bilirubin $(9-30 \mathrm{mmol} / \mathrm{L})$ and conjugated bilirubin $(6.5-12 \mathrm{mmol} / \mathrm{L})$ of the treated mice also increased significantly $(p>0.05)$ when compared with the normoglycaemic mice. Total protein decreased significantly for both extract-treated groups while urea and creatinine levels were unaffected. Phytochemical screening of Azadirachta indica seed oil extracts revealed the presence of alkaloids, flavonoids, saponnins, steroids, terpenes and tannins. The result of this study shows that the methanolic and ethanolic extracts of A. indica possess hypoglyceamic properties but may be hepatotoxic.
\end{abstract}

Key Words: Azadirachta indica, Diabetes, Normoglycaemic, Hypoglycaemic, Hepatotoxic.

\section{INTRODUCTION}

The World Health Organization defines traditional medicine as the sum total of knowledge, skills and practices based on the theories, beliefs and experiences indigenous to different cultures (1). These benefits are used to maintain health, as well as to prevent, diagnose, improve or treat physical and mental illnesses. Plants and their constituents have a long history of use in certain systems of traditional medicine, and are the sources of important drugs such as atropine, codeine, digoxin, morphine, quinine and vincristine (2). Use of herbal medicines in developing countries is on increase because of poverty and the assumption that it has mild side effects. The active principles of some of these plant products have been isolated and characterized, and their mechanisms of action are still under study (3). The complexity of herbal and botanical preparations is that they are not pure compounds, and it is a function of the traditionally-held belief that the synergistic combination of several active principles in some herbal preparations is responsible for their beneficial effects (4). Diabetes mellitus is a metabolic disorder in which a person has a high level of blood sugar as a result of deficiency of insulin or insensitivity of insulin receptors. The disease has been treated over the years with diet and herbs until the advent of modern pharmaceuticals but even with that, there is still a rampant increase of this autoimmune response disease (5). Owing to this, searches for a new and better anti-hyperglyceamic drug is being carried out especially in the use of herbs because of the perception that there is a lower incidence of adverse reaction to plant preparations compared to synthetic pharmaceuticals and reduced costs of plant preparations (6). Modern medicines, despite offering a variety of effective treatment options, can have several adverse effects.

Neem (Azadirachta indica) is a tropical evergreen tree native majorly to Indian subcontinent and Subsaharan African. The tree belongs to the Meliceae family and grows rapidly in the tropic and semi tropic climate. All parts of the plant- leaves, bark, flower, fruits, seed and root have been used in the treatment of ailments such as of fever, and eczema and are known to possess antimicrobial and anti-inflammatory activities (7). The aqueous leaf extract of Azadirachta indica when orally fed to rats produced hypoglycemia in normal 
and diabetic rats (8). Azadirachta indica seed has a very high concentration of oil and the oil is used to treat cases of diabetes in rural Hausa community of Northern Nigeria. This research was therefore designed to evaluate the effectiveness or otherwise of Azadirachta indica seed oil in the treatment of diabetes in order to justify its folkoric use in Northern part of Nigeria. It is hoped that those who suffer from this universal disorder will get relief from it with mild adverse effects without insulin injections.

\subsection{Plant Sample}

\section{MATERIALS AND METHODS}

Fresh Azadirachta indica kernels were collected under a Neem tree within the premises of Federal University of Technology, Minna, by May, 2012 and authenticated at the Herbarium section of the Department of Biological Sciences, Federal University of Technology, Minna, Nigeria.

\subsection{Experimental Animals}

Healthy Swiss albino mice of either sexes and of six weeks old, weighing between 20-30g were obtained from Vom, in Jos, Plateau State, Nigeria. They were housed under standard conditions in Departmental laboratory with free access to commercial grower feed and water.

\subsection{Sample Preparation}

The kernels were washed and air dried in the laboratory for twelve days and then depulped to obtain the seeds. The seeds were further homogenized with mortar and pestle to obtain a fine powder.

\subsection{Methanol and Ethanol Extraction of powdered Seed}

About One hundred and twelve grams (112.5g) of the dried powder seed was weighed in two places, one was extracted with $700 \mathrm{ml}$ of methanol solution while the other with $600 \mathrm{ml}$ of ethanol solution using the soxhlet extraction method at temperatures of $65^{\circ} \mathrm{C}$ and $75^{\circ} \mathrm{C}$ respectively. A rotary evaporator was used to evaporate the solvents from the extracts to obtain the oil. The oil was kept in a well labeled sterile bottle and stored in refrigerator till ready for use.

\subsection{Phytochemical Screening}

Phytochemical tests for bioactive constituents were carried out on the oil extracts of Azadirachta indica seed using standard laboratory procedures as described by (9).

\subsection{Grouping of Animals}

The design consisted of twenty mice, sixteen were rendered diabetic and four normoglycaemic. The mice were divided into five groups of four mice each. Group one was normoglyceamic; groups two and three were diabetic and were treated with $200 \mathrm{mg} / \mathrm{kg}$ bodyweight methanolic and ethanolic extracts. Group four mice were diabetic untreated (but administered $10 \mathrm{ml} / \mathrm{kg}$ bodyweight of normal saline). Group five mice were given $200 \mathrm{mg} / \mathrm{kg}$ bodyweight standard drug.

\subsection{Induction of Diabetes in Mice}

$300 \mathrm{mg} / \mathrm{kg}$ bodyweight of alloxan solution was administered intraperitoneally to animals in the diabetic group after an overnight fast of twelve hours with access to only water to make them more susceptible to developing diabetes (10). All the experimental animals were allowed free access to food and water after induction and maintained under the same laboratory conditions. The glucose levels of the diabetic induced mice were checked after seventy-two hours in order to establish the diabetic status.

\subsection{Administration of Extracts}

Animals in Groups two and three were orally administered respective extract $(200 \mathrm{mg} / \mathrm{kg}$ bodyweight $)$ twice daily in a twelve hour cycle ( 9 am and $9 \mathrm{pm}$ ), while group four animals were administered normal saline $(10 \mathrm{ml} / \mathrm{kg}$ bodyweight). The group five mice were administered standard drug $(200 \mathrm{mg} / \mathrm{kg}$ bodyweight). Group one was normoglycaemic. In vivo measurement of blood glucose was carried out for seven days with blood obtained from the tail vein of the mice using One Touch Glucometer.

\subsection{Determination of Hematological Parameters}

At the end of one week, the animals were fasted for 12 hours and anaesthetized under chloroform vapour and sacrificed. The blood obtained by cardiac puncture was collected into syringes and taken to the laboratory for biochemical assays. Total and conjugated bilirubin of the test samples were determined using TECO Laboratory kit. Alkaline Phosphatase, kinetic determination of Alanine aminotransaminase and aspartate transaminase, total protein, urea, electrolytes, and creatinine were determined by using respective TECO diagnostic kit for each parameter. PCV (Haematocrit) was determined by. 


\section{RESULTS}

The methanol extract yielded $30.27 \mathrm{~g}(27.0 \%)$, while the ethanol extract yielded $27.4 \mathrm{~g}(24.5 \%)$.

\subsection{Phytochemical Content}

Table 1 shows the phytochemical compositions of the methanolic and ethanolic extracts of $A$.indica seed oil. The methanolic extract contains alkaloids, cardiac glycosides, terpenes, steroids, and flavonoids in high presence, while the ethanolic extract contains only steroids in high presence. Both extracts had moderate presence of phenols, with only ethanol extract having alkaloids, tannins, cardiac glycosides, terpenes, and flavonoids in moderate presence.

Table 1: Phytochemical Components of Extracts of Azadirachta indica Seed Oil.

\begin{tabular}{lcc}
\hline Component & Methanol extract & Ethanol extracts \\
\hline Saponin & ++ & + \\
Alkaloids & +++ & ++ \\
Phenols & ++ & ++ \\
Tannins & + & ++ \\
Cardiac glycosides & +++ & ++ \\
Terpenes & +++ & ++ \\
Steroids & +++ & +++ \\
Flavonoid & +++ & ++ \\
Anthraguinones & - & - \\
\hline +++ Highly present & & \\
++ Moderately present & & \\
$\quad+\quad$ Present & &
\end{tabular}

\subsection{Percentage Packed Cell Volume (PCV)}

Figure-1 shows the values of percentage packed cell volume for the tests and control groups. The group that was administered ethanol extract (EETM) had the \% pcv increased, while the \% pcv of diabetic untreated (DU) group decreased when both are compared with the mice in normoglycaemic (NMG) group.

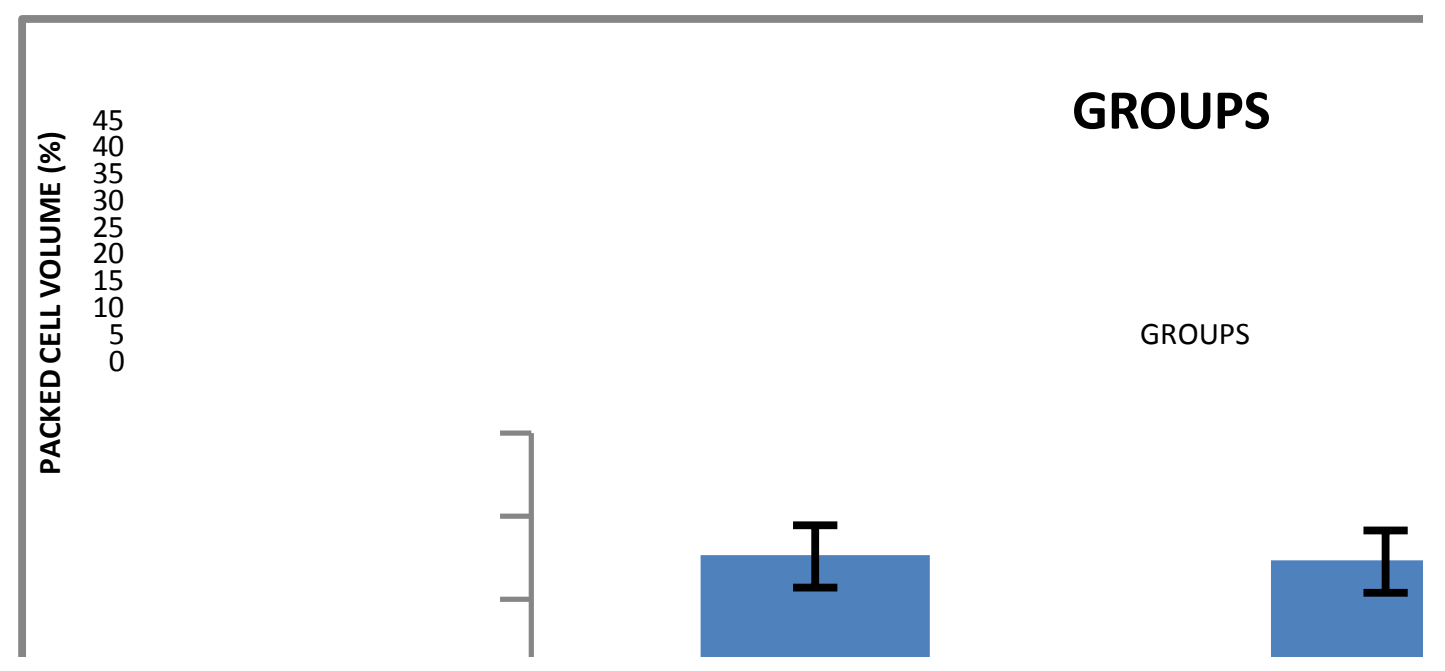

Figure 1: Packed Cell Volume Variation after Treatment

- $\quad$ NMG : Normoglyceamic

- D.U: Diabetic Untreated

- METFORMIN : Standard drug

- METM : Methanolic extract treated mice

- $\quad$ EETM : Ethanolic extract treated mice 


\subsection{Blood Glucose Levels of Treated and Untreated mice}

Figure -2 shows that the mice treated with the standard drug (metmorfin), ethanol (EETM), and methanol (METM) extracts all had their blood glucose decreased in descending order during the period of treatments (seven days), with METM having the highest activity. The blood glucose of diabetic untreated mice (DU) continued to increase until two of the mice in the group died two days before the termination of the experiment (in seven days).

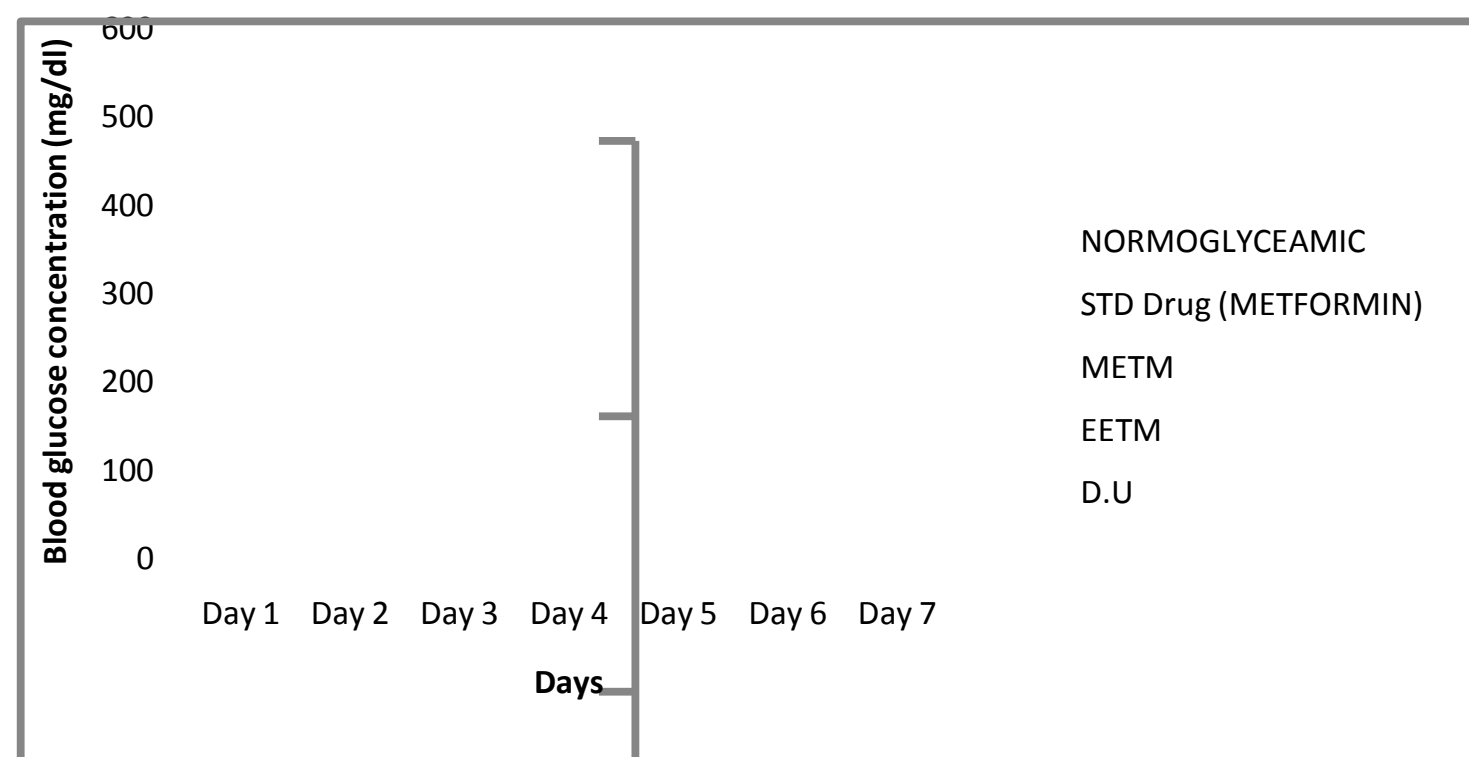

Figure- 2: Blood Glucose Level (1-7 Days)

- METM : Methanolic extract treated mice

- EETM : Ethanolic extract treated mice

- D.U: Diabetic Untreated

\subsection{Serum Levels of Biochemical Parameters}

Figure -3 shows the serum levels of some biochemical parameters in the test and control groups. The test groups all had high values of both conjugated (C.B), and total bilirubin (T.B) with the exception of the animals in D.U group. Total protein was high in STD, and D.U groups, while urea was high in METM and EETM groups, but low in STD group.

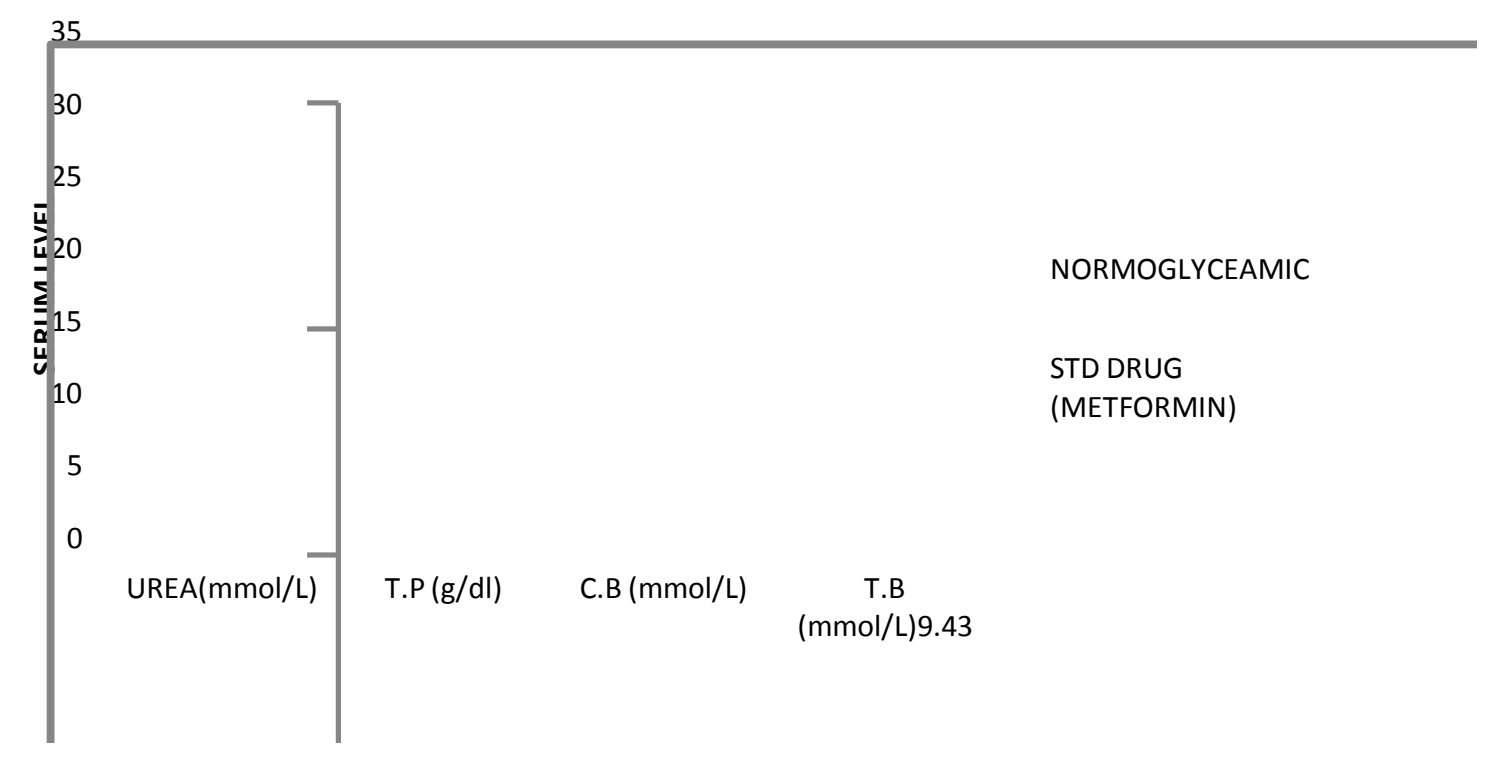

Figure- 3: Serum Levels of Biochemical Parameters 
- $\quad$ C.B : Conjugated Bilirubin

- T.B : Total Bilirubin

- D.U : Diabetic Untreated

- T.P : Total Protein

- $\quad$ METM : Methanolic extract treated mice

- $\quad$ EETM : Ethanolic extract treated mic

\subsection{Activities of Enzymes and Level of Creatinine}

The serum activities of aspartate aminotransferase (AST), alanine aminotransferase (ALT), alkaline phosphatase (ALP), and the level of serum creatinine are shown on figure-4. The activities of AST, ALT, and ALP all increased in STD, METM, and EETM groups, while ALT, and ALP activities decreased in DU group, with an increase in the activity of AST in DU group. The activity of AST is highest in METM, EETM, and DU groups. Serum creatinine decreased in all the four groups when compared with the normoglycaemic group.

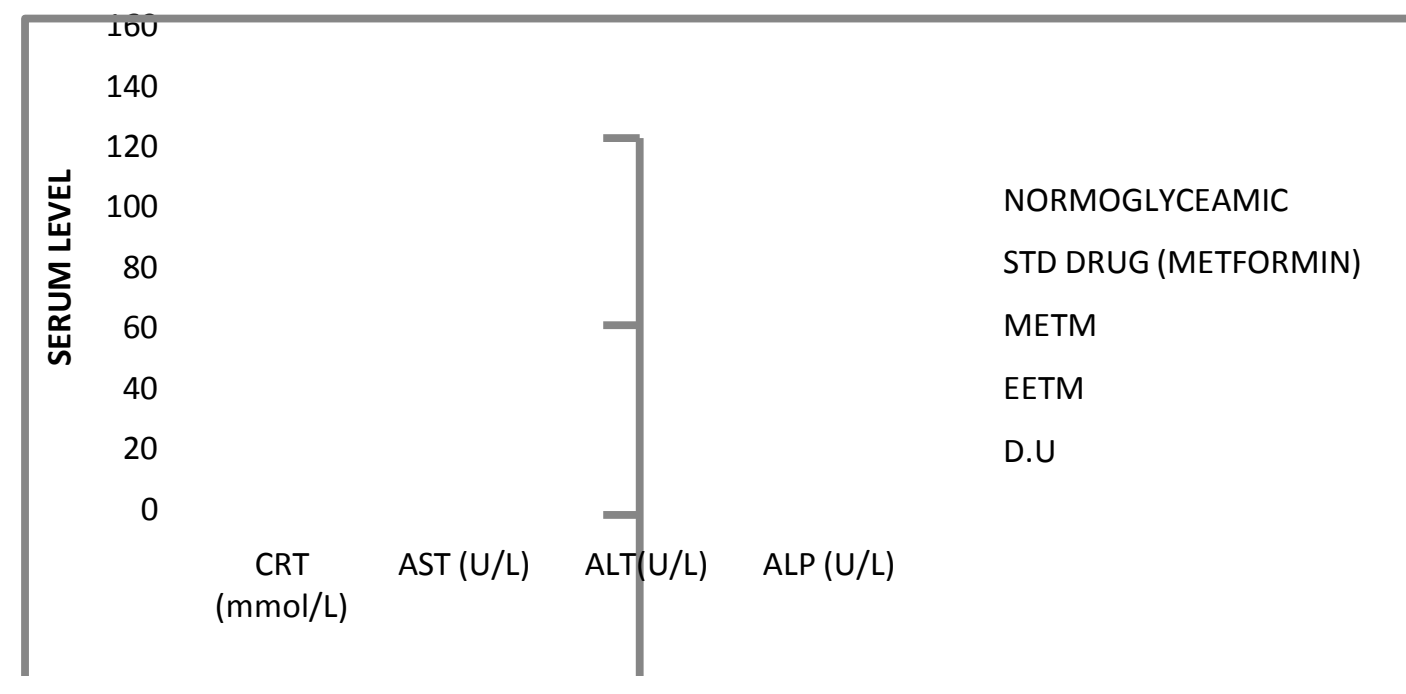

Figure- 4. Serum levels of Creatinine (CRT), Aspartate aminotransferase (AST), Alanine aminotransferase (ALT) and Alkaline phosphatase (ALP).

- D.U : Diabetic Untreated

- $\quad$ METM :Methanolic extract treated mice

- $\quad$ EETM :Ethanolic extract treated mice

\section{DISCUSSION AND CONCLUSION}

Phytochemical screening of methanol and ethanol extracts of Azadirachta indica seed oil revealed the presence of alkaloids, saponins, steroids, terpenes, tannins, phenols, cardiac glycosides and flavonoids (table 1). The evidence of these significant phytochemicals may be responsible for the efficacy and medicinal use of the plant (11). The bioactive components of the oil derived from crushing the seed has a very wide spectrum of action in medicine, and they aid in the treatment of diabetes by opposing autoimmune response, helping the beta cells to produce insulin (12). According (13), administration of the leaf extracts to laboratory rats caused decreasing of blood glucose levels. (14) also reported that leaf extracts produced an approximately 35\% reduction in blood glucose levels in normal and streptozocin diabetic induced rats. Oral administration of both methanolic and ethanolic extracts of Azadirachta indica seed oil to diabetic mice produced a significant $(\mathrm{p}<$ 0.05 ) reduction in the blood glucose concentrations (figure 1). Similar observation has been reported in rats following the administration of Azadirachta indica leaf extract (15). The hypoglyceamic effect of the methanolic extract was more pronounced than the ethanolic extract and the standard drug (figure 1). This may be due to the ability of the methanol solvent to extract more phytoconstituents. No death or obvious signs of toxicity was observed throughout the period of treatment.Urea is the major waste product of protein metabolism, and urea levels are used to detect diseases and disorders that affect the kidney e.g, acute kidney failure. Ammonia released during deamination is removed from the blood by its conversion into urea. In serious liver disease, ammonia accumulates in the blood, and the glomerular filtration of urea is low (see figure 3 ). 
Urea varies inversely with the rate of excretion and directly with protein intake. Creatinine is a chemical waste molecule generated from muscle metabolism, and is found to be a very reliable indicator of kidney function. It's retention in the blood is as a result of impairment of the normal functioning of the kidney and rise is due to poor clearance of creatinine by the kidneys. Result obtained showed that there was no significant difference in the serum level creatinine in the extracts treated mice when compared with the normoglyceamic mice except for the standard drug treated group (low creatinine level). This result is in line with the work reported by (16), where administration of crude aqueous extract of neem leaves had no significant changes on the urea and creatinine levels. This may indicate that there was probably impairment in the urea cycle or as a result of dehydration (17).

Serum levels of the enzymes found in two major organs of the body (the liver and kidney) which are vital to healthy living were also determined. The serum levels of Alanine aminotransferase (AST) and Aspartate aminotransferase (AST) of both extracts and standard drug treated mice showed a significant $(\mathrm{p}<0.05)$ increase compared to the normoglyceamic. This agrees with the work of Rahiya (18). Alanine aminotransferase is an excellent marker enzyme found in the liver. Injury to the liver results in release of the enzyme into the blood. An increase in the level of this enzyme often means that liver disease such as cirrhosis is present or the liver has been exposed to toxic substances. ALT is more liver specific than AST. Rise in levels of ALT is usually followed by a rise in the level of AST (19).

Alkaline phosphatase is a protein found in all body tissues with particularly high amounts in the liver. The serum levels of alkaline phosphatase significantly increased in both extract treated animals compared to the normoglyceamic mice. Higher than normal may be due to biliary obstruction leading to its diffusion into the blood. This is in line with the result obtained by (20). Lower than normal may be due to malnutrition although, lower than normal level is not common. However, the ALP level of the methanolic extract treated group was higher than that of the ethanolic extract and the standard drug treated groups. The diabetic untreated group maintained the normal range.

Total protein consists of albumin and globulin. The liver to a large extent synthesizes albumin and some fraction of globulin. Low level of total protein was observed in both extract treated animals compared to the normoglyceamic and standard drug treated animals. This agrees with work of (21), where the effect of neem gold was observed on total protein. The diabetic untreated group was within range. This low level may be due to impaired liver damage or malabsorption.

Bilirubin is the break down product of normal heme catabolism. It is excreted in bile and urine. Total bilirubin is the lipid soluble form of bilirubin that circulates in loose association with the plasma protein while conjugated bilirubin is water soluble and synthesized in the liver from indirect bilirubin. The serum level of bilirubin of the extract treated animals increased significantly compared to the normoglyceamic mice. This may be as a result of rapid hemolysis of red blood cells and the hepatic cells cannot excrete bilirubin as rapidly as it is formed. The plasma concentration of free bilirubin was high, the rate of formation of urobilinogen in the intestine was increased, and much of it was reabsorbed into the blood. The rapid release of bilirubin into the blood may also be as a result of obstruction of bile ducts or damage to the liver cells, so that even the usual amounts of bilirubin cannot be excreted into the gastrointestinal tracts. The elevated level observed may indicate liver damage or disease (22).

Packed cell volume is the ratio of the volume occupied by packed red blood cell to the volume of the whole blood as measured by a hematocrit. An individual with hematocrit 30 is said to have $30 \%$ of its blood volume made up of cells. Below 40 and 35 for male and female respectively is interpreted to imply aneamia, but above that is interpreted polycythemia. There was no significant difference between the normoglyceamic, standard drug treated group and the groups treated with the extracts. This agrees with the findings of Ngure et al. (23). However, a decrease observed in the diabetic untreated group implies that the mice were aneamic and aneamia may be caused by poor nutrition and disease conditions.

Methanol and ethanol seed oil extracts of Azadirachta indica have the ability to reduce diabetes in mice although they may have hepatotoxic effects (especially the methanolic extract). The methanol extract seemed to have more glucose lowering potential than the ethanolic extract and the standard drug. The glucose lowering effect explains the rationale for use of the Azadrachita indica seed oil in traditional folklore. The oil extracts could at least in part stimulate inslin production and glucose utilization like the standard drug -metmorfin, Oil seed of $A$. indica can therefore be a veritable and cheaper substitute for conventional drugs since it is easily obtainable. 


\section{REFERENCES}

[1] World Health Organization (WHO) (2008). Department of Noncommunicable Diseases Surveillance. Definition, Diagnosis and classification of Diabetes Mellitus and its Complications. Pp 620.

[2] Yadav M., Vikram N.K., and Sharma D (2010). Complimentary and comparative study on hypoglycaemic and antihyperglycaemic activity of various extracts of Eugenia jambolana seed, Gymmema sylvestre and Trigomella foenum graecum seeds in rats. Applied Biochemistry and Biotechnology. 160: (8) 238-400.

[3] .Sudha P., Remya R., Smita Z., Shobha B., and Ameet R. K (2011). Evaluation of traditional Indian antidiabetic medicinal plants for human pancreatic amylase inhibitory effect in vivo. American journal of Biochemistry and Biotechnology. Vol 3: 173-175.

[4] Chee F., Savkin A.V., Fernando T.L., and Nahavan S (2005).Insulin injection control for glucose regulation in diabetic patients. Biomedical Engineering 52: 1625-1631.

[5] Lenzen S (2008). The mechanisms of alloxan and streptozotocin induced diabetes. Diabetologia :216-226

[6] Samuel K., Kwasi B., Michael A., and Jerome D (2011). Toxicological analysis and effect neem tree extracts in an organism. European Journal of Experimental Biology 1(2): 160-171.

[7] Subapriya R., and Nagini S (2005). Medicinal properties of neem leaves: a review. Current medical and chemical anticancer agents. 5: 149-156.

[8] Alimat, M., Sharma, D., and Ansari, B (2012). Effect of lamda-cyhalothrin and neem gold in the gill liver and ovary of zebra fish. Journal of veterinary pathology. 20: 19-25.

[9] Sofowora A (2006). Medicinal plants and traditional medicine in Africa, Spectrum Books Ltd, Ibadan, Nigeria, pp 150.

[10] Cowell M.D (2008). Focus on Diagnosis: Type 2 diabetes mellitus. Pediatrics Review. 29 289-292

[11] Wilcox, M., and Chamberlain J (2004). Azadirachta indica in traditional medicinal plant of malria. Bora ratton CRC Press PP 99121.

[12] Schmutterer, H (2002). The neem tree: Source of unique natural products for integrated pest management, medicine, industry and other purposes, VCH, Weinheim, Germany, pp. 1-6.

[13] Sinniah D., Schwartz P. H., Mitchell R. A., and Arcinue E.L (1985). Investigations of an animal model of a Reye-like syndrome caused by Margosa oil. Pediatary Research. 19 (12): 1346-1355.

[14] Halim, E.M (2000). Lowering of blood sugar by water extract of Azadirachta indica and Abroma augusta in diabetic rats. Indian Journal of Experimental Biology, 41: 636-640.

[15] Halim, E. M. and Ali, H. (2002). Reversal of diabetic retinopathy in streptozotocin induced diabetic rats using traditional indian anti-diabetic plant, Azadirachta indica. Indian Journal of Clinical Biochemmistry 17(2):115-123

[16] Samuel K., Kwasi B., Michael A., and Jerome D (2011). Toxicological analysis and effect neem tree extracts in an organism. European Journal of Experimental Biology 1(2): 160-171.

[17] Neelesh M., Sanjay J., and Sapna M (2010). Antidiabetic potential of medicinal plants. Drug Research 67(2), 113-118.

[18] Rahiya, S (2005). Studies on the effects of Azadirachta indica leaf extract on pathology of experimental fowl typhoid in broiler chicken. Indian Journal of Veterenary Pathology. 29:151-152.

[19] Moss, D., and Henderson, A (1999). Text book of clinical enzymology. Serum chemical biomarkers of cardiac injury for non clinical safety testing. Pp 617-721.

[20] Brown G., Neath I., and Chater, N (2007). A temporary ratio model of memory, Psychological review 114: 539-576.

[21] Alimat, M., Sharma, D., and Ansari, B (2012). Effect of lamda-cyhalothrin and neem gold in the gill liver and ovary of zebra fish. Journal of veterinary pathology. 20: 19-25.

[22] Liu Y., Li P., Lu J., Oger J., and Tetzlaff, W (2008). Bilirubin posseses powerful immunomodulatory activity and suppresses experimental autoinnune encephalomyelitis. Journal of immunology 181(3): 1887-97.

[23] Ngure R., Bosire 0., Stephen M., William W., and Ronald G (2009). Anti-trypanosomal effects of Azadirachta indica extracts on trypanosoma brucei and rhodosiense-infected mice. Eastern Journal of Medicine 14:2-9. 